\title{
Random Polygons and Optimal Extrapolation Estimates of $\pi$
}

\author{
Shasha Wang ${ }^{1}$, Wen-Qing $\mathrm{Xu}^{2}$, Jitao Liu ${ }^{1, *}$ \\ ${ }^{1}$ School of Mathematics, Beijing University of Technology, China; \\ ${ }^{2}$ Department of Mathematics and Statistics, California State University, Long Beach, USA
}

\begin{abstract}
We construct optimal extrapolation estimates of $\pi$ based on random polygons generated by $n$ independent points uniformly distributed on a unit circle in $\mathbb{R}^{2}$. While the semiperimeters and areas of these random $n$-gons converge to $\pi$ almost surely and are asymptotically normal as $n \rightarrow \infty$, in this paper we develop various extrapolation processes to further accelerate such convergence. By simultaneously considering the random $n$-gons and suitably constructed random $2 n$-gons and then optimizing over functionals of the semiperimeters and areas of these random polygons, we derive several new estimates of $\pi$ with faster convergence rates. These extrapolation improvements are also shown to be asymptotically normal as $n \rightarrow \infty$.
\end{abstract}

Keywords Random Approximations of $\pi$, Extrapolation, Convergence, Stochastic Optimization, Central Limit Theorems

AMS 2010 subject classifications Primary 60D05, 60F05; Secondary 05C80, 65C20

DOI: $10.19139 /$ soic-2310-5070-1193

\section{Introduction}

Given a convex set $K \subseteq \mathbb{R}^{d}$, the stochastic properties of the convex hull $K_{n}$ generated by $n$ independent points chosen at random in $K$ have been studied by many authors. For example, for $K \subset \mathbb{R}^{2}$ bounded and convex, and the random points uniformly distributed over $K$, Rényi and Sulanke [13, 14] derived limiting expressions for the expected values of the number of vertices, the perimeter and the area of $K_{n}$. In the case of a unit disk in $\mathbb{R}^{2}$, Hsing [7] further proved that the asymptotic variance of the area $A_{n}$ of the convex hull $K_{n}$ is of the order $n^{-5 / 3}$ and satisfies the central limit theorem $n^{5 / 6}\left(A_{n}-\mathbb{E}\left(A_{n}\right)\right) \stackrel{\mathcal{L}}{\rightarrow} \mathcal{N}\left(0, \sigma^{2}\right)$ where $\sigma^{2}=\lim _{n \rightarrow \infty} n^{5 / 3} \operatorname{Var}\left(A_{n}\right)$ is a positive constant and the notation $\stackrel{\mathcal{L}}{\rightarrow}$ denotes convergence in distribution [3, 5, 15]. In [17], Vu further extended these results to general dimensions $d \geq 2$ and established central limit theorems for the volume and the number of all lowerdimensional faces of the random polytope $K_{n}$. As an example of unbounded sets, Hueter [8] considered $n$ random points independently and normally distributed in the plane $\mathbb{R}^{2}$ and showed that the area $A_{n}$ of the corresponding convex hull satisfies the central limit theorem $\left(A_{n}-2 \pi \log n\right) / \log n \stackrel{\mathcal{L}}{\rightarrow} \mathcal{N}\left(0,2 \pi^{3 / 2}\right)$ as $n \rightarrow \infty$.

More recently, Bélisle [2] (see also [19,21]) studied the case when the points are independently and uniformly randomly selected on the unit circle in $\mathbb{R}^{2}$. In such cases, the resulting convex hull is an inscribed random $n$ gon which can be obtained by simply connecting all adjacent vertices on the circle. Additionally, a random circumscribing $n$-gon may also be constructed which is tangent to the circle at each of the random vertices [20]. Then the semiperimeter $S_{n}$ and the area $A_{n}$ of the random inscribed $n$-gon and the semiperimeter (and area) $S_{n}^{\prime}$ of the random circumscribing $n$-gon all converge to $\pi$ with probability 1 (abbre. as w.p.1) as $n \rightarrow \infty$ with

*Correspondence to: Jitao Liu (Email: jtliu @ bjut.edu.cn). School of Mathematics, Faculty of Science, Beijing University of Technology, 100 Pingleyuan, Chaoyang District, Beijing, China (100124).

ISSN 2310-5070 (online) ISSN 2311-004X (print)

Copyright (C) 2021 International Academic Press 
$S_{n}=\pi+n^{-1+\delta} o(1), A_{n}=\pi+n^{-1+\delta} o(1)$ and $S_{n}^{\prime}=\pi+n^{-1+\delta} o(1)$ for any $\delta>0$, where each $o(1)$ represents a random variable that converges to 0 w.p. 1 as $n \rightarrow \infty$. Moreover, the distributions of $S_{n}, A_{n}$ and $S_{n}^{\prime}$ are also asymptotically normal with $n^{5 / 2}\left(S_{n}-\left(\pi-\pi^{3} / n^{2}\right)\right) \stackrel{\mathcal{L}}{\rightarrow} \mathcal{N}\left(0,10 \pi^{6}\right), n^{5 / 2}\left(A_{n}-\left(\pi-4 \pi^{3} / n^{2}\right)\right) \stackrel{\mathcal{L}}{\rightarrow} \mathcal{N}\left(0,160 \pi^{6}\right)$ and $n^{5 / 2}\left(S_{n}^{\prime}-\left(\pi+2 \pi^{3} / n^{2}\right)\right) \stackrel{\mathcal{L}}{\rightarrow} \mathcal{N}\left(0,40 \pi^{6}\right)$. See also [18] for extensions of these convergence results to cases when the vertices are not independently or not uniformly distributed on the circle.

In the special case when the vertices happen to be equally spaced on the circle, such cyclic polygons become regular polygons inscribed in or circumscribed about the circle with $S_{n}=n \sin \frac{\pi}{n}=\pi-\frac{\pi^{3}}{6 n^{2}}+$ $\frac{\pi^{5}}{120 n^{4}}-\frac{\pi^{5}}{5040 n^{6}}+\cdots=\pi+O\left(n^{-2}\right), A_{n}=\frac{1}{2} n \sin \frac{2 \pi}{n}=\pi-\frac{2 \pi^{3}}{3 n^{2}}+\frac{2 \pi^{5}}{15 n^{4}}-\frac{4 \pi^{5}}{315 n^{6}}+\cdots=\pi+O\left(n^{-2}\right)$ and $S_{n}^{\prime}=$ $n \tan \frac{\pi}{n}=\pi+\frac{\pi^{3}}{3 n^{2}}+\frac{2 \pi^{5}}{15 n^{4}}+\frac{17 \pi^{7}}{315 n^{6}}+\cdots=\pi+O\left(n^{-2}\right)$ for large $n$. Since Archimedes, these geometric quantities have provided famous approximations of $\pi[1,10,16]$. By further applying extrapolation methods $[9,12]$ which go back to Huygens, it is easy to see that the weighted average $\mathcal{Y}_{n}=\frac{2}{3} S_{n}+\frac{1}{3} S_{n}^{\prime}=\pi+\frac{\pi^{5}}{20 n^{4}}+\cdots=\pi+O\left(n^{-4}\right)$ generates a far more accurate estimate of $\pi$ than either $S_{n}$ or $S_{n}^{\prime}$ alone. In fact, by including also $A_{n}$, it is possible to further kill the error terms at the order $O\left(n^{-4}\right)$ to obtain $\mathcal{Z}_{n}=\frac{16}{15} S_{n}-\frac{1}{5} A_{n}+\frac{2}{15} S_{n}^{\prime}=\pi+\frac{\pi^{7}}{105 n^{6}}+$ $\cdots=\pi+O\left(n^{-6}\right)$. In [19, 20], such linear combinations have been shown to hold also for random cyclic polygons with $\mathcal{Y}_{n}=\frac{2}{3} S_{n}+\frac{1}{3} S_{n}^{\prime}=\pi+n^{-3+\delta} o(1)$ and $\mathcal{Z}_{n}=\frac{16}{15} S_{n}-\frac{1}{5} A_{n}+\frac{2}{15} S_{n}^{\prime}=\pi+n^{-5+\delta} o(1)$. Moreover, $\mathcal{Y}_{n}$ and $\mathcal{Z}_{n}$ are also asymptotically normal with $n^{9 / 2}\left(\mathcal{Y}_{n}-\left(\pi+6 \pi^{5} / n^{4}\right)\right) \stackrel{\mathcal{L}}{\rightarrow} \mathcal{N}\left(0,8136 \pi^{10}\right)$ and $n^{13 / 2}\left(\mathcal{Z}_{n}-(\pi+\right.$ $\left.\left.48 \pi^{7} / n^{6}\right)\right) \stackrel{\mathcal{L}}{\rightarrow} \mathcal{N}\left(0,7792128 \pi^{14}\right)$.

Note that the original Archimedean approach also incorporates an important doubling process of the number of vertices of the regular polygons with the famous geometric-harmonic-mean relations $1 / S_{n}+1 / S_{n}^{\prime}=2 / S_{2 n}^{\prime}$, $S_{n} S_{2 n}^{\prime}=S_{2 n}^{2}$ providing the key tool for efficient computations of the semiperimeters $S_{2 n}$ and $S_{2 n}^{\prime}$ of the corresponding regular $2 n$-gons directly from the semiperimeters $S_{n}$ and $S_{n}^{\prime}$ of the preceding regular $n$-gons. With each iteration of this doubling process, the approximation errors decrease roughly by a factor of $1 / 4$, that is, $S_{2 n}-$ $\pi \approx \frac{1}{4}\left(S_{n}-\pi\right)$ and $S_{2 n}^{\prime}-\pi \approx \frac{1}{4}\left(S_{n}^{\prime}-\pi\right)$. Similarly, for $\mathcal{Y}_{n}=\frac{2}{3} S_{n}+\frac{1}{3} S_{n}^{\prime}$ and $\mathcal{Z}_{n}=\frac{16}{15} S_{n}-\frac{1}{5} A_{n}+\frac{2}{15} S_{n}^{\prime}$, we have $\mathcal{Y}_{2 n}-\pi \approx \frac{1}{16}\left(\mathcal{Y}_{n}-\pi\right), \mathcal{Z}_{2 n}-\pi \approx \frac{1}{64}\left(\mathcal{Z}_{n}-\pi\right)$. By applying again extrapolation techniques, we may obtain several improved estimates through, for example, $\mathcal{X}_{n}=\frac{4}{3} S_{2 n}-\frac{1}{3} S_{n}=\pi-\frac{\pi^{5}}{480 n^{4}}+\cdots, \mathcal{Y}_{n}^{*}=\frac{16}{15} \mathcal{Y}_{2 n}-\frac{1}{15} \mathcal{Y}_{n}=$ $\frac{32}{45} S_{2 n}+\frac{16}{45} S_{2 n}^{\prime}-\frac{2}{45} S_{n}-\frac{1}{45} S_{n}^{\prime}=\pi-\frac{\pi^{7}}{1120 n^{6}}+\cdots$, and $\mathcal{Z}_{n}^{*}=\frac{64}{63} \mathcal{Z}_{2 n}-\frac{1}{63} \mathcal{Z}_{n}=-\frac{208}{945} S_{n}+\frac{1}{315} A_{n}-\frac{2}{945} S_{n}^{\prime}+$ $\frac{1024}{945} S_{2 n}+\frac{128}{945} S_{2 n}^{\prime}=\pi-\frac{\pi^{9}}{30240 n^{8}}+\cdots$. In fact, by optimizing over all linear combinations of $S_{n}, A_{n}, S_{n}^{\prime}$ and $S_{2 n}$, $A_{2 n}$ (which equals $S_{n}$ ), $S_{2 n}^{\prime}$, we can further obtain $\mathcal{W}_{n}=-\frac{976}{3465} S_{n}+\frac{3}{385} A_{n}-\frac{2}{3465} S_{n}^{\prime}+\frac{4096}{3465} S_{2 n}+\frac{64}{693} S_{2 n}^{\prime}=$ $\pi-\frac{\pi^{11}}{221760 n^{10}}+\cdots$.

In this paper, we aim to extend these extrapolation estimates to the more interesting case of random inscribed and circumscribed polygons. However, unlike the case of regular polygons, the process to double the number of vertices of a random polygon is more complicated and how this is done may affect the corresponding optimal extrapolation estimates. Such an effort was first made in [19] where the author proposed three different approaches for the doubling process, namely, independent doubling, equal bisection and random bisection respectively, albeit for the relatively easy case of inscribed polygons only. The first approach simply uses an independent set of $2 n$ vertices independently and uniformly distributed on the circle. The second approach retains the original $n$ random vertices and at the same time inserts $n$ new vertices by equally bisecting each of the resulting $n$ arcs. The third approach offers a kind of hybrid of the above two approaches where instead of the midpoints, a new random vertex is inserted on each of the $n$ arcs separated by the original random vertices. Here we follow the same doubling approaches, but include also random circumscribing polygons so that we can take full advantage of the estimates made available by the doubling process to obtain even faster rates of convergence than those derived in [19].

The equal bisection approach turns out to be the closest to the classical Archimedean case. As is shown in [19], in terms of linear combinations of $S_{n}$ and $S_{2 n}$, exactly the same expression $\mathcal{X}_{n}=$ $\frac{4}{3} S_{2 n}-\frac{1}{3} S_{n}$ satisfies $\mathcal{X}_{n}=\pi+n^{-3+\delta} o(1)$ for any $\delta>0$ with $n^{9 / 2}\left(\mathcal{X}_{n}-\left(\pi-\pi^{5} / n^{4}\right)\right) \stackrel{\mathcal{L}}{\rightarrow} \mathcal{N}\left(0,113 \pi^{10} / 8\right)$. Similarly, for $\mathcal{Y}_{n}=\frac{2}{3} S_{n}+\frac{1}{3} S_{n}^{\prime}$ and $\mathcal{Z}_{n}=\frac{16}{15} S_{n}-\frac{1}{5} A_{n}+\frac{2}{15} S_{n}^{\prime}$, we can derive the extrapolation improvements $\mathcal{Y}_{n}^{*}=\frac{16}{15} \mathcal{Y}_{2 n}-\frac{1}{15} \mathcal{Y}_{n}=-\frac{2}{45} S_{n}-\frac{1}{45} S_{n}^{\prime}+\frac{32}{45} S_{2 n}+\frac{16}{45} S_{2 n}^{\prime}, \mathcal{Z}_{n}^{*}=\frac{64}{63} \mathcal{Z}_{2 n}-\frac{1}{63} \mathcal{Z}_{n}=-\frac{208}{945} S_{n}+\frac{1}{315} A_{n}-\frac{2}{945} S_{n}^{\prime}+$ $\frac{1024}{945} S_{2 n}+\frac{128}{945} S_{2 n}^{\prime} \quad$ with $\mathcal{Y}_{n}^{*}=\pi+n^{-5+\delta} o(1), \quad \mathcal{Z}_{n}^{*}=\pi+n^{-7+\delta} o(1) \quad$ and $\quad n^{13 / 2}\left(\mathcal{Y}_{n}^{*}-\left(\pi-9 \pi^{7} /\left(2 n^{6}\right)\right)\right) \stackrel{\mathcal{L}}{\rightarrow}$ 
$\mathcal{N}\left(0,136971 \pi^{14} / 2\right), n^{17 / 2}\left(\mathcal{Z}_{n}^{*}-\left(\pi-12 \pi^{9} / n^{8}\right)\right) \stackrel{\mathcal{L}}{\rightarrow} \mathcal{N}\left(0,6989472 \pi^{18}\right)$. On the other hand, by directly optimizing over all linear combinations of the quantities $S_{n}, A_{n}, S_{n}^{\prime}, S_{2 n}, S_{2 n}^{\prime}$ (again with $A_{2 n}=S_{n}$ ), we can obtain the same optimal estimate $\mathcal{W}_{n}=-\frac{976}{3465} S_{n}+\frac{3}{385} A_{n}-\frac{2}{3465} S_{n}^{\prime}+\frac{4096}{3465} S_{2 n}+\frac{64}{693} S_{2 n}^{\prime}=\pi+n^{-9+\delta} o(1)$ with $n^{21 / 2}\left(\mathcal{W}_{n}-\right.$ $\left.\left(\pi-180 \pi^{11} / n^{10}\right)\right) \stackrel{\mathcal{L}}{\rightarrow} \mathcal{N}\left(0,22852044000 \pi^{22}\right)$. See Theorems 4.1-4.2 below for details.

However, for the independent doubling and random bisection approaches, due to additional sources of the randomness, it becomes much harder (or impossible) to achieve the same level of extrapolation improvements. For example, when combining $S_{n}^{\prime}$ and $S_{2 n}^{\prime}$, no matter how we choose the coefficients, there is no way to cancel the leading order error terms in $S_{n}^{\prime}$ and $S_{2 n}^{\prime}$ to improve upon $\mathcal{X}_{n}^{\prime}=\alpha S_{2 n}^{\prime}+\beta S_{n}^{\prime}=\pi+n^{-1+\delta} o(1)$. Due to this, we choose instead to minimize the bias in its leading error term. This yields $\alpha=4 / 3$ and $\beta=-1 / 3$ for independent doubling with $n^{5 / 2}\left(\mathcal{X}_{n}^{\prime}-\pi\right) \stackrel{\mathcal{L}}{\rightarrow} \mathcal{N}\left(0,20 \pi^{6} / 3\right)$, and $\alpha=2, \beta=-1$ for the random bisection approach with $n^{5 / 2}\left(\mathcal{X}_{n}^{\prime}-\pi\right) \stackrel{\mathcal{L}}{\rightarrow} \mathcal{N}\left(0,16 \pi^{6}\right)$. Similar conclusions may also be drawn for $\mathcal{Y}_{n}^{*}=\alpha \mathcal{Y}_{2 n}+\beta \mathcal{Y}_{n}$ and $\mathcal{Z}_{n}^{*}=\alpha \mathcal{Z}_{2 n}+\beta \mathcal{Z}_{n}$. See Theorems 3.1-3.2 and 5.1-5.2 for details.

The remainder of the paper is organized as follows. First we state some useful preliminary results in Section 2. Then we present the three doubling processes and establish the corresponding optimal extrapolation estimates respectively in Sections 3, 4 and 5.

\section{Preliminaries}

\subsection{Random divisions of the unit interval}

Let $n \geq 2$ and $X_{0}:=0<X_{1}<\cdots<X_{n-1}<X_{n}:=1$ be the order statistics of $n-1$ independent and uniformly distributed random points on the unit interval. It is well-known [4, 11] that the $n$ spacings $X_{i}-X_{i-1}, i=$ $1,2, \cdots, n$, are identically distributed with common probability density function (PDF) $f(x)=(n-1)(1-x)^{n-2}$ for $0<x<1$, and for any $i \neq j$, the joint PDF of $X_{i}-X_{i-1}$ and $X_{j}-X_{j-1}$ is given by $f(x, y)=(n-1)(n-$ $2)(1-x-y)^{n-3}$ for $x>0, y>0$, and $x+y<1$.

Let $k \in \mathbb{N}$ and $\mathcal{D}_{n, k}=\sum_{i=1}^{n}\left|X_{i}-X_{i-1}\right|^{k}$. Then it is easy to compute that $\mathbb{E}\left(\mathcal{D}_{n, k}\right)=k ! n ! /(n+k-1) ! \approx$ $k ! / n^{k-1}, \operatorname{Var}\left(\mathcal{D}_{n, k}\right) \approx\left\{(2 k) !-\left(1+k^{2}\right)(k !)^{2}\right\} / n^{2 k-1}$ for large $n$. Furthermore, we have

Lemma 2.1 (Asymptotic convergence of $\mathcal{D}_{n, k}[2,19]$ )

For any $k \in \mathbb{N}$ and $\delta>0$, it holds that

1. $n^{k-1-\delta} \mathcal{D}_{n, k} \rightarrow 0$ in probability, and $n^{k-2-\delta} \mathcal{D}_{n, k} \rightarrow 0$ w.p. 1 as $n \rightarrow \infty$,

2. $\sqrt{n}\left(n^{k-1} \mathcal{D}_{n, k}-k !\right) \stackrel{\mathcal{L}}{\rightarrow} \mathcal{N}\left(0, \sigma_{k}^{2}\right)$ where $\sigma_{k}^{2}=(2 k) !-\left(1+k^{2}\right)(k !)^{2}$.

\subsection{Random cyclic polygons}

Given $n$ independent random points uniformly distributed on the unit circle, we label them $P_{i}=\left(\cos \theta_{i}, \sin \theta_{i}\right)$, $0 \leq i \leq n$ in counterclockwise direction where $\theta_{0}<\theta_{1}<\cdots<\theta_{n-1}<\theta_{n}=\theta_{0}+2 \pi$ and $P_{n}$ represents the same point as $P_{0}$. Without loss of generality, we assume $\theta_{0}=0$. A further rescaling $\theta_{i}=2 \pi X_{i}$ then yields $0=$ $X_{0}<X_{1}<\cdots<X_{n-1}<X_{n}=1$, which amounts to a random division of $(0,1)$ by $n-1$ independent random points uniformly distributed over $(0,1)$. By connecting these consecutive vertices, we obtain a random inscribed $n$-gon $P_{1} P_{2} \cdots P_{n}$ with semiperimeter and area given respectively by $S_{n}=\sum_{i=1}^{n} \sin \pi\left(X_{i}-X_{i-1}\right), A_{n}=$ $\frac{1}{2} \sum_{i=1}^{n} \sin 2 \pi\left(X_{i}-X_{i-1}\right)$.

By using Lemma 2.1 and the Taylor approximation $\left|\sin x-\sum_{i=1}^{m}(-1)^{i-1} \frac{1}{(2 i-1) !} x^{2 i-1}\right| \leq \frac{1}{(2 m+1) !}|x|^{2 m+1}$ of the sine function for any $m \in \mathbb{N}$ and $x \in \mathbb{R}$, it is known that [19]

$$
\begin{aligned}
& S_{n}=\sum_{j=1}^{m} \frac{(-1)^{j-1}}{(2 j-1) !} \pi^{2 j-1} \mathcal{D}_{n, 2 j-1}+n^{-(2 m-1)+\delta} o(1), \\
& A_{n}=\sum_{j=1}^{m} \frac{(-1)^{j-1} 2^{2 j-2}}{(2 j-1) !} \pi^{2 j-1} \mathcal{D}_{n, 2 j-1}+n^{-(2 m-1)+\delta} o(1) .
\end{aligned}
$$


On the other hand, the tangent lines through any two consecutive vertices $P_{i-1}$ and $P_{i}$ intersect outside the circle at a point $Q_{i}=\left(\frac{\cos \pi\left(X_{i}+X_{i-1}\right)}{\cos \pi\left(X_{i}-X_{i-1}\right)}, \frac{\sin \pi\left(X_{i}+X_{i-1}\right)}{\cos \pi\left(X_{i}-X_{i-1}\right)}\right)$. By connecting these points consecutively, we can similarly obtain a random circumscribing $n$-gon $Q_{1} Q_{2} \cdots Q_{n}$ which is tangent to the circle at each $P_{i}$ with both semiperimeter and area now given by $S_{n}^{\prime}=\sum_{i=1}^{n} \tan \frac{\theta_{i}-\theta_{i-1}}{2}=\sum_{i=1}^{n} \tan \pi\left(X_{i}-X_{i-1}\right)$.

Note that if $\theta_{i}-\theta_{i-1} \geq \pi$ for some $i$, or equivalently, $\Delta_{n}:=\max _{1 \leq i \leq n}\left|X_{i}-X_{i-1}\right| \geq 1 / 2$, such random circumscribing polygons actually do not exist (when $\Delta_{n}=1 / 2$ ) or fall completely outside the circle (when $\Delta_{n}>$ $1 / 2$ ). Also, due to the singularity of $\tan x$ at $x=\pi / 2$, even if $\Delta_{n}<1 / 2, S_{n}^{\prime}$ may become unbounded when $\Delta_{n}$ gets close to $1 / 2$. Nevertheless, by using a cut-off technique for the Taylor series $\tan x=\sum_{i=1}^{\infty} \frac{B_{2 i}(-4)^{i}\left(1-4^{i}\right)}{(2 i) !} x^{2 i-1}=$ $x+\frac{1}{3} x^{3}+\frac{2}{15} x^{5}+\frac{17}{315} x^{7}+\frac{62}{2835} x^{9}+\cdots$ where $B_{j}$ is the $j$ th Bernoulli number, together with Lemma 2.1 and the exponential decay property of the tail probability $\operatorname{Pr}\left(\Delta_{n} \geq t\right)$ for any $t \in(0,1)$ as $n \rightarrow \infty$, it is still possible to obtain a similar asymptotic expansion for $S_{n}^{\prime}$ for any $m \geq 1$ [20]:

$$
S_{n}^{\prime}=\sum_{j=1}^{m} \frac{B_{2 j}(-4)^{j}\left(1-4^{j}\right)}{(2 j) !} \pi^{2 j-1} \mathcal{D}_{n, 2 j-1}+n^{-(2 m-1)+\delta} o(1) .
$$

In particular, for $m=1$, we see that $S_{n}, A_{n}, S_{n}^{\prime} \rightarrow \pi$ w.p.1 as $n \rightarrow \infty$. Furthermore, as in the case of regular polygons, by using linear combinations of $S_{n}, A_{n}, S_{n}^{\prime}$, we can obtain the following extrapolation improvements.

Lemma $2.2([19,20])$

Let $\mathcal{X}_{n}=\frac{4}{3} S_{n}-\frac{1}{3} A_{n}, \mathcal{Y}_{n}=\frac{2}{3} S_{n}+\frac{1}{3} S_{n}^{\prime}$ and $\mathcal{Z}_{n}=\frac{16}{15} S_{n}-\frac{1}{5} A_{n}+\frac{2}{15} S_{n}^{\prime}$. Then for any $\delta>0$, it holds that $\mathcal{X}_{n}=$ $\pi+n^{-3+\delta} o(1), \mathcal{Y}_{n}=\pi+n^{-3+\delta} o(1), \mathcal{Z}_{n}=\pi+n^{-5+\delta} o(1)$ and $n^{9 / 2}\left(\mathcal{X}_{n}-\left(\pi-4 \pi^{5} / n^{4}\right)\right) \stackrel{\mathcal{L}}{\rightarrow} \mathcal{N}\left(0,3616 \pi^{10}\right)$, $n^{9 / 2}\left(\mathcal{Y}_{n}-\left(\pi+6 \pi^{5} / n^{4}\right)\right) \stackrel{\mathcal{L}}{\rightarrow} \mathcal{N}\left(0,8136 \pi^{10}\right), n^{13 / 2}\left(\mathcal{Z}_{n}-\left(\pi+48 \pi^{7} / n^{6}\right)\right) \stackrel{\mathcal{L}}{\rightarrow} \mathcal{N}\left(0,7792128 \pi^{14}\right)$.

\section{Independent Doubling of Random Cyclic Polygons}

To extend the classical extrapolation methods based on Archimedean polygons [1,9] to the case of random cyclic polygons, we use similar ideas in [19] to double the sides of a random $n$-gon so that the semiperimeters and areas of the random $n$-gons and $2 n$-gons may be combined to generate higher order accurate estimates of $\pi$. We start in this section with the relatively easy case of independent doubling, that is, the random $2 n$-gons are generated by $2 n$ independent points uniformly distributed on the unit circle in $\mathbb{R}^{2}$, independent of how the vertices of the preceding random $n$-gons are chosen. Thus we have two independent sets of random divisions of $(0,1)$, one for the $n$-gons by $0=X_{0}<X_{1}<\cdots<X_{n-1}<X_{n}=1$, and one for the $2 n$-gons by $0=Y_{0}<Y_{1}<\cdots<$ $Y_{2 n-1}<Y_{2 n}=1$. The semiperimeters and areas of the newly constructed random $2 n$-gons can now be expressed as $S_{2 n}=\sum_{i=1}^{2 n} \sin \pi\left(Y_{i}-Y_{i-1}\right), A_{2 n}=\frac{1}{2} \sum_{i=1}^{2 n} \sin 2 \pi\left(Y_{i}-Y_{i-1}\right), S_{2 n}^{\prime}=\sum_{i=1}^{2 n} \tan \pi\left(Y_{i}-Y_{i-1}\right)$.

With the availability of the additional estimates $S_{2 n}, A_{2 n}$ and $S_{2 n}^{\prime}$, we may apply extrapolation techniques to derive improved estimates for $\pi$. By replacing $n$ with $2 n$ in Lemma 2.2, similar convergence estimates clearly also hold for $\mathcal{X}_{2 n}=\frac{4}{3} S_{2 n}-\frac{1}{3} A_{2 n}, \mathcal{Y}_{2 n}=\frac{2}{3} S_{2 n}+\frac{1}{3} S_{2 n}^{\prime}$, and $\mathcal{Z}_{2 n}=\frac{16}{15} S_{2 n}-\frac{1}{5} A_{2 n}+\frac{2}{15} S_{2 n}^{\prime}$. Here we focus on the construction of further extrapolation improvements, making full use of the semiperimeters and areas of both the $n$-gons and the $2 n$-gons.

We start with linear combinations of $S_{n}^{\prime}$ and $S_{2 n}^{\prime}$ in the form of $\mathcal{X}_{n}^{\prime}=\alpha S_{2 n}^{\prime}+\beta S_{n}^{\prime}$ where $\alpha, \beta \in \mathbb{R}$. Clearly for $\mathcal{X}_{n}^{\prime} \rightarrow \pi$ as $n \rightarrow \infty$, it is necessary (and sufficient) that $\alpha+\beta=1$. Taking $m=2$ in (3) yields $S_{n}^{\prime}=$ $\pi+\frac{1}{3} \pi^{3} \mathcal{D}_{n, 3}+n^{-3+\delta} o(1)$ and $S_{2 n}^{\prime}=\pi+\frac{1}{3} \pi^{3} \mathcal{D}_{2 n, 3}+n^{-3+\delta} o(1)$, and hence $\mathcal{X}_{n}^{\prime}=\pi+\frac{1}{3} \pi^{3}\left(\alpha \mathcal{D}_{2 n, 3}+\beta \mathcal{D}_{n, 3}\right)+$ $n^{-3+\delta} o(1)$ where $\mathcal{D}_{n, 3}=\sum_{i=1}^{n}\left|X_{i}-X_{i-1}\right|^{3}$ and $\mathcal{D}_{2 n, 3}=\sum_{i=1}^{2 n}\left|Y_{i}-Y_{i-1}\right|^{3}$ are independent of each other. As a result, no matter how we choose the coefficients $\alpha$ and $\beta$, there is no way to eliminate the leading order error term containing $\alpha \mathcal{D}_{2 n, 3}+\beta \mathcal{D}_{n, 3}$ to achieve $\mathcal{X}_{n}^{\prime}=\pi+n^{-3+\delta} o(1)$. In view of this, we turn to minimize instead the bias in $\mathcal{X}_{n}^{\prime}$ which implies $\mathbb{E}\left(\alpha \mathcal{D}_{2 n, 3}+\beta \mathcal{D}_{n, 3}\right)=0$, or equivalently, $\alpha+4 \beta=0$. Together with $\alpha+\beta=1$, this yields $\alpha=4 / 3, \beta=-1 / 3$, and hence $\mathcal{X}_{n}^{\prime}=\pi+\frac{1}{3} \pi^{3} \mathcal{D}_{n, 3}^{*}+n^{-3+\delta} o(1)$ where $\mathcal{D}_{n, 3}^{*}=\frac{4}{3} \mathcal{D}_{2 n, 3}-\frac{1}{3} \mathcal{D}_{n, 3}$.

By Lemma 2.1, $\mathcal{X}_{n}^{\prime}$ clearly satisfies $n^{1-\delta}\left(\mathcal{X}_{n}^{\prime}-\pi\right) \rightarrow 0$ w.p.1 for any $\delta>0$. Also, due to the independence of $\mathcal{D}_{2 n, 3}$ and $\mathcal{D}_{n, 3}$, it holds that $n^{5 / 2} \mathcal{D}_{n, 3}^{*}=\frac{4}{3} n^{5 / 2}\left(\mathcal{D}_{2 n, 3}-3 ! /\left(4 n^{2}\right)\right)-\frac{1}{3} n^{5 / 2}\left(\mathcal{D}_{n, 3}-3 ! / n^{2}\right) \stackrel{\mathcal{L}}{\rightarrow} \mathcal{N}(0,60)$. 
By using Slutsky's theorem [6, 15] (with $0<\delta \leq 1 / 2)$, we then obtain $n^{5 / 2}\left(\mathcal{X}_{n}^{\prime}-\pi\right) \stackrel{\mathcal{L}}{\rightarrow} \mathcal{N}\left(0,20 \pi^{6} / 3\right)$. Similarly, by combining $S_{n}$ with $S_{2 n}^{\prime}$, or $A_{n}$ with $S_{2 n}^{\prime}$, we can obtain $\mathcal{Y}_{n}^{\prime}=\frac{2}{3} S_{2 n}^{\prime}+\frac{1}{3} S_{n}=\pi+\frac{1}{6} \pi^{3} \mathcal{D}_{n, 3}^{*}+$ $n^{-3+\delta} o(1), \quad \mathcal{Z}_{n}^{\prime}=\frac{8}{9} S_{2 n}^{\prime}+\frac{1}{9} A_{n}=\pi+\frac{2}{9} \pi^{3} \mathcal{D}_{n, 3}^{*}+n^{-3+\delta} o(1)$ with $n^{5 / 2}\left(\mathcal{Y}_{n}^{\prime}-\pi\right) \stackrel{\mathcal{L}}{\rightarrow} \mathcal{N}\left(0,5 \pi^{6} / 3\right), n^{5 / 2}\left(\mathcal{Z}_{n}^{\prime}-\right.$ $\pi) \stackrel{\mathcal{L}}{\rightarrow} \mathcal{N}\left(0,80 \pi^{6} / 27\right)$. Thus, we obtain the following asymptotic results of $\mathcal{X}_{n}^{\prime}, \mathcal{Y}_{n}^{\prime}$ and $\mathcal{Z}_{n}^{\prime}$.

Theorem 3.1

Let $\mathcal{X}_{n}^{\prime}=\frac{4}{3} S_{2 n}^{\prime}-\frac{1}{3} S_{n}^{\prime}, \mathcal{Y}_{n}^{\prime}=\frac{2}{3} S_{2 n}^{\prime}+\frac{1}{3} S_{n}$ and $\mathcal{Z}_{n}^{\prime}=\frac{8}{9} S_{2 n}^{\prime}+\frac{1}{9} A_{n}$. Then for any $\delta>0$, it holds that $\mathcal{X}_{n}^{\prime}=\pi+$ $n^{-1+\delta} o(1), \mathcal{Y}_{n}^{\prime}=\pi+n^{-1+\delta} o(1), \mathcal{Z}_{n}^{\prime}=\pi+n^{-1+\delta} o(1)$ and $n^{5 / 2}\left(\mathcal{X}_{n}^{\prime}-\pi\right) \stackrel{\mathcal{L}}{\rightarrow} \mathcal{N}\left(0,20 \pi^{6} / 3\right), n^{5 / 2}\left(\mathcal{Y}_{n}^{\prime}-\pi\right) \stackrel{\mathcal{L}}{\rightarrow}$ $\mathcal{N}\left(0,5 \pi^{6} / 3\right), n^{5 / 2}\left(\mathcal{Z}_{n}^{\prime}-\pi\right) \stackrel{\mathcal{L}}{\rightarrow} \mathcal{N}\left(0,80 \pi^{6} / 27\right)$.

Next, by further combining $\mathcal{Y}_{n}=\frac{2}{3} S_{n}+\frac{1}{3} S_{n}^{\prime}$ and $\mathcal{Y}_{2 n}=\frac{2}{3} S_{2 n}+\frac{1}{3} S_{2 n}^{\prime}$, we obtain $\mathcal{Y}_{n}^{*}=\alpha \mathcal{Y}_{2 n}+\beta \mathcal{Y}_{n}=\pi+$ $\frac{1}{20} \pi^{5}\left(\alpha \mathcal{D}_{2 n, 5}+\beta \mathcal{D}_{n, 5}\right)+n^{-5+\delta} o(1)$ where $\alpha, \beta \in \mathbb{R}$ and $\alpha+\beta=1$. Again, since there is no way to eliminate the term $\alpha \mathcal{D}_{2 n, 5}+\beta \mathcal{D}_{n, 5}$, we choose $\alpha, \beta$ such that $\mathbb{E}\left(\alpha \mathcal{D}_{2 n, 5}+\beta \mathcal{D}_{n, 5}\right)=0$. This yields $\alpha=16 / 15, \beta=-1 / 15$, and hence $\mathcal{Y}_{n}^{*}=\frac{16}{15} \mathcal{Y}_{2 n}-\frac{1}{15} \mathcal{Y}_{n}=-\frac{2}{45} S_{n}-\frac{1}{45} S_{n}^{\prime}+\frac{32}{45} S_{2 n}+\frac{16}{45} S_{2 n}^{\prime}=\pi+\frac{1}{300} \pi^{5} \mathcal{D}_{n, 5}^{*}+n^{-5+\delta} o(1)$ where $\mathcal{D}_{n, 5}^{*}=$ $16 \mathcal{D}_{2 n, 5}-\mathcal{D}_{n, 5}$ satisfies $n^{9 / 2} \mathcal{D}_{n, 5}^{*}=-n^{9 / 2}\left(\mathcal{D}_{n, 5}-5 ! / n^{4}\right)+16 n^{9 / 2}\left(\mathcal{D}_{2 n, 5}-5 ! /\left(16 n^{4}\right)\right) \stackrel{\mathcal{L}}{\rightarrow} \mathcal{N}(0,4881600)$.

Finally, by combining $\mathcal{Z}_{n}=\frac{16}{15} S_{n}-\frac{1}{5} A_{n}+\frac{2}{15} S_{n}^{\prime}$ and $\mathcal{Z}_{2 n}=\frac{16}{15} S_{2 n}-\frac{1}{5} A_{2 n}+\frac{2}{15} S_{2 n}^{\prime}$, we get $\mathcal{Z}_{n}^{*}=$ $\frac{64}{63} \mathcal{Z}_{2 n}-\frac{1}{63} \mathcal{Z}_{n}=-\frac{16}{945} S_{n}+\frac{1}{315} A_{n}-\frac{2}{945} S_{n}^{\prime}+\frac{1024}{945} S_{2 n}-\frac{64}{315} A_{2 n}+\frac{128}{945} S_{2 n}^{\prime}=\pi+\frac{1}{6615} \pi^{\top} \mathcal{D}_{n, 7}^{*}+n^{-7+\delta} o(1)$ where $\mathcal{D}_{n, 7}^{*}=64 \mathcal{D}_{2 n, 7}-\mathcal{D}_{n, 7} \quad$ satisfies $\quad n^{13 / 2} \mathcal{D}_{n, 7}^{*}=-n^{13 / 2}\left(\mathcal{D}_{n, 7}-7 ! / n^{6}\right)+64 n^{13 / 2}\left(\mathcal{D}_{2 n, 7}-7 ! /(2 n)^{6}\right) \stackrel{\mathcal{L}}{\rightarrow}$ $\mathcal{N}(0,128862316800)$. Therefore, it follows that

\section{Theorem 3.2}

Let $\quad \mathcal{Y}_{n}^{*}=-\frac{2}{45} S_{n}-\frac{1}{45} S_{n}^{\prime}+\frac{32}{45} S_{2 n}+\frac{16}{45} S_{2 n}^{\prime} \quad$ and $\quad \mathcal{Z}_{n}^{*}=-\frac{16}{945} S_{n}+\frac{1}{315} A_{n}-\frac{2}{945} S_{n}^{\prime}+\frac{1024}{945} S_{2 n}-\frac{64}{315} A_{2 n}+$ $\frac{128}{945} S_{2 n}^{\prime}$. Then for any $\delta>0$, it holds that $\mathcal{Y}_{n}^{*}=\pi+n^{-3+\delta} o(1), \mathcal{Z}_{n}^{*}=\pi+n^{-5+\delta} o(1)$ and $n^{9 / 2}\left(\mathcal{Y}_{n}^{*}-\pi\right) \stackrel{\mathcal{L}}{\rightarrow}$ $\mathcal{N}\left(0,1356 \pi^{10} / 25\right), n^{13 / 2}\left(\mathcal{Z}_{n}^{*}-\pi\right) \stackrel{\mathcal{L}}{\rightarrow} \mathcal{N}\left(0,432896 \pi^{14} / 147\right)$.

\section{Bisection of the Random $n$-gon}

Next, we take the bisection approach to construct random $2 n$-gons, namely by inserting a new vertex $P_{i-1 / 2}=$ $\left(\cos \theta_{i-1 / 2}, \sin \theta_{i-1 / 2}\right)$ exactly half way between any two consecutive vertices $P_{i-1}$ and $P_{i}$ of the existing random $n$-gon, where $\theta_{i-1 / 2}=\left(\theta_{i-1}+\theta_{i}\right) / 2$. With the same rescaling $\theta_{i-1 / 2}=2 \pi X_{i-1 / 2}$, we obtain $0=X_{0}<X_{1 / 2}<$ $X_{1}<\cdots<X_{n-1}<X_{n-1 / 2}<X_{n}=1$. While this may also be viewed as a random division of $(0,1)$, the $2 n-1$ points $X_{1 / 2}, X_{1}, \cdots, X_{n-1}, X_{n-1 / 2}$ do not correspond to the order statistics of $2 n-1$ independent points uniformly distributed on $(0,1)$, and thus the corresponding random $2 n$-gons do not behave the same as random $2 n$ gons generated directly by $2 n$ independent points uniformly distributed on the unit circle. Nevertheless, these newly constructed random $2 n$-gons turn out to be the closest to the Archimedean approach. For notational convenience, their semiperimeters and areas are again denoted by $S_{2 n}, A_{2 n}, S_{2 n}^{\prime}$. Then we have $S_{2 n}=2 \sum_{i=1}^{n} \sin \frac{\pi\left(X_{i}-X_{i-1}\right)}{2}$, $S_{2 n}^{\prime}=2 \sum_{i=1}^{n} \tan \frac{\pi\left(X_{i}-X_{i-1}\right)}{2}$ with $A_{2 n}=\sum_{i=1}^{n} \sin \pi\left(X_{i}-X_{i-1}\right)=S_{n}$. By using the Taylor expansions for $\tan x$ and $\sin x$, similar to (1), (2) and (3), we have, for any $m \geq 1$,

$$
\begin{aligned}
& S_{2 n}=\sum_{j=1}^{m}(-1)^{j-1} \frac{\pi^{2 j-1}}{2^{2 j-2}(2 j-1) !} \mathcal{D}_{n, 2 j-1}+n^{-(2 m-1)+\delta} o(1), \\
& A_{2 n}=\sum_{j=1}^{m}(-1)^{j-1} \frac{\pi^{2 j-1}}{(2 j-1) !} \mathcal{D}_{n, 2 j-1}+n^{-(2 m-1)+\delta} o(1), \\
& S_{2 n}^{\prime}=\sum_{j=1}^{m} \frac{B_{2 j}(-4)^{j}\left(1-4^{j}\right)}{(2 j) !} \frac{\pi^{2 j-1}}{2^{2 j-2}} \mathcal{D}_{n, 2 j-1}+n^{-(2 m-1)+\delta} o(1) .
\end{aligned}
$$

By combining $S_{n}, A_{n}$ with $S_{2 n}$ or $A_{2 n}$, it is known [19] that $\mathcal{X}_{n}=\frac{4}{3} S_{2 n}-\frac{1}{3} S_{n}, \mathcal{Y}_{n}=\frac{4}{3} A_{2 n}-\frac{1}{3} A_{n}, \mathcal{Z}_{n}=$ $\frac{64}{45} S_{2 n}-\frac{4}{9} S_{n}+\frac{1}{45} A_{n}$ provide improved approximations of $\pi$. Here, by including also $S_{n}^{\prime}$ or $S_{2 n}^{\prime}$, we aim to construct additional higher-order extrapolation estimates of $\pi$.

First, we consider $\mathcal{X}_{n}^{\prime}=\alpha S_{2 n}^{\prime}+\beta S_{n}^{\prime}$ with $\alpha+\beta=1$. By using $S_{2 n}^{\prime}=\pi+\frac{1}{12} \pi^{3} \mathcal{D}_{n, 3}+\frac{1}{120} \pi^{5} \mathcal{D}_{n, 5}+$ $n^{-5+\delta} o(1) \quad$ and $\quad S_{n}^{\prime}=\pi+\frac{1}{3} \pi^{3} \mathcal{D}_{n, 3}+\frac{2}{15} \pi^{5} \mathcal{D}_{n, 5}+n^{-5+\delta} o(1), \quad$ we have $\mathcal{X}_{n}^{\prime}=\pi+\frac{1}{12} \pi^{3}(\alpha+4 \beta) \mathcal{D}_{n, 3}+$ 
$\frac{1}{120} \pi^{5}(\alpha+16 \beta) \mathcal{D}_{n, 5}+n^{-5+\delta} o(1)$. Note that by setting $\alpha+4 \beta=0$, it is now possible to eliminate the leading error term involving $\mathcal{D}_{n, 3}$ in $\mathcal{X}_{n}^{\prime}$. Together with $\alpha+\beta=1$, this yields $\alpha=4 / 3, \beta=-1 / 3$, and thus $\mathcal{X}_{n}^{\prime}=\frac{4}{3} S_{2 n}^{\prime}-$ $\frac{1}{3} S_{n}^{\prime}=\pi-\frac{1}{30} \pi^{5} \mathcal{D}_{n, 5}+n^{-5+\delta} o(1)$ with $n^{9 / 2}\left(\mathcal{X}_{n}^{\prime}-\left(\pi-4 \pi^{5} / n^{4}\right)\right)=-\frac{1}{30} \pi^{5} n^{9 / 2}\left(\mathcal{D}_{n, 5}-5 ! / n^{4}\right)+n^{-1 / 2+\delta_{o}}(1)$. By Lemma 2.1, we have $n^{9 / 2}\left(\mathcal{D}_{n, 5}-5 ! / n^{4}\right) \stackrel{\mathcal{L}}{\rightarrow} \mathcal{N}(0,3254400)$. Then by applying Slutsky's theorem, we obtain $n^{9 / 2}\left(\mathcal{X}_{n}^{\prime}-\left(\pi-4 \pi^{5} / n^{4}\right)\right) \stackrel{\mathcal{L}}{\rightarrow} \mathcal{N}\left(0,3616 \pi^{10}\right)$ as $n \rightarrow \infty$.

Similarly, we may combine $S_{2 n}^{\prime}$ and $S_{n}$ or $S_{2 n}^{\prime}$ and $A_{n}$ to obtain $\mathcal{Y}_{n}^{\prime}=\frac{2}{3} S_{2 n}^{\prime}+\frac{1}{3} S_{n}=\pi+$ $\frac{1}{120} \pi^{5} \mathcal{D}_{n, 5}+n^{-5+\delta} o(1)$ and $\mathcal{Z}_{n}^{\prime}=\frac{8}{9} S_{2 n}^{\prime}+\frac{1}{9} A_{n}=\pi+\frac{1}{45} \pi^{5} \mathcal{D}_{n, 5}+n^{-3+\delta} o(1)$ with $n^{9 / 2}\left(\mathcal{Y}_{n}^{\prime}-\left(\pi+\pi^{5} / n^{4}\right)\right) \stackrel{\mathcal{L}}{\rightarrow}$ $\mathcal{N}\left(0,226 \pi^{10}\right)$ and $n^{9 / 2}\left(\mathcal{Z}_{n}^{\prime}-\left(\pi+8 \pi^{5} /\left(3 n^{4}\right)\right)\right) \stackrel{\mathcal{L}}{\rightarrow} \mathcal{N}\left(0,14464 \pi^{10} / 9\right)$. We note that these estimates for $\mathcal{X}_{n}^{\prime}, \mathcal{Y}_{n}^{\prime}$ and $\mathcal{Z}_{n}^{\prime}$ are stronger than those in Theorem 3.1 for the case of independent doubling.

Theorem 4.1

Let $\mathcal{X}_{n}^{\prime}=\frac{4}{3} S_{2 n}^{\prime}-\frac{1}{3} S_{n}^{\prime}, \mathcal{Y}_{n}^{\prime}=\frac{2}{3} S_{2 n}^{\prime}+\frac{1}{3} S_{n}$ and $\mathcal{Z}_{n}^{\prime}=\frac{8}{9} S_{2 n}^{\prime}+\frac{1}{9} A_{n}$. Then for any $\delta>0$, it holds that $\mathcal{X}_{n}^{\prime}=$ $\pi+n^{-3+\delta} o(1), \mathcal{Y}_{n}^{\prime}=\pi+n^{-3+\delta} o(1), \mathcal{Z}_{n}^{\prime}=\pi+n^{-3+\delta} o(1)$ and $n^{9 / 2}\left(\mathcal{X}_{n}^{\prime}-\left(\pi-4 \pi^{5} / n^{4}\right)\right) \stackrel{\mathcal{L}}{\rightarrow} \mathcal{N}\left(0,3616 \pi^{10}\right)$, $n^{9 / 2}\left(\mathcal{Y}_{n}^{\prime}-\left(\pi+\pi^{5} / n^{4}\right)\right) \stackrel{\mathcal{L}}{\rightarrow} \mathcal{N}\left(0,226 \pi^{10}\right), n^{9 / 2}\left(\mathcal{Z}_{n}^{\prime}-\left(\pi+8 \pi^{5} /\left(3 n^{4}\right)\right)\right) \stackrel{\mathcal{L}}{\rightarrow} \mathcal{N}\left(0,14464 \pi^{10} / 9\right)$.

Next, to eliminate the error term containing $\mathcal{D}_{n, 5}$, we combine $\mathcal{Y}_{n}=\frac{2}{3} S_{n}+\frac{1}{3} S_{n}^{\prime}$ and $\mathcal{Y}_{2 n}=\frac{2}{3} S_{2 n}+$ $\frac{1}{3} S_{2 n}^{\prime}$ to obtain $\mathcal{Y}_{n}^{*}=\frac{16}{15} \mathcal{Y}_{2 n}-\frac{1}{15} \mathcal{Y}_{n}=-\frac{2}{45} S_{n}-\frac{1}{45} S_{n}^{\prime}+\frac{32}{45} S_{2 n}+\frac{16}{45} S_{2 n}^{\prime}=\pi-\frac{1}{1120} \pi^{7} \mathcal{D}_{n, 7}+n^{-7+\delta} o(1)$. By further combining $\mathcal{Z}_{n}=\frac{16}{15} S_{n}-\frac{1}{5} A_{n}+\frac{2}{15} S_{n}^{\prime}$ and $\mathcal{Z}_{2 n}=\frac{16}{15} S_{2 n}-\frac{1}{5} A_{2 n}+\frac{2}{15} S_{2 n}^{\prime}$, we also have $\mathcal{Z}_{n}^{*}=\frac{64}{63} \mathcal{Z}_{2 n}-$ $\frac{1}{63} \mathcal{Z}_{n}=-\frac{208}{945} S_{n}+\frac{1}{315} A_{n}-\frac{2}{945} S_{n}^{\prime}+\frac{1024}{945} S_{2 n}+\frac{128}{945} S_{2 n}^{\prime}=\pi-\frac{1}{30240} \pi^{9} \mathcal{D}_{n, 9}+n^{-9+\delta} o(1)$. Finally, like in the Archimedean case, by optimizing over all linear combinations of $S_{n}, A_{n}, S_{n}^{\prime}$ and $S_{2 n}, S_{2 n}^{\prime}$ (again with $\left.A_{2 n}=S_{n}\right)$, we can obtain $\mathcal{W}_{n}=-\frac{976}{3465} S_{n}+\frac{3}{385} A_{n}-\frac{2}{3465} S_{n}^{\prime}+\frac{4096}{3465} S_{2 n}+\frac{64}{693} S_{2 n}^{\prime}=\pi-\frac{1}{221760} \pi^{11} \mathcal{D}_{n, 11}+$ $n^{-11+\delta} O(1)$. Therefore, we obtain the following theorem.

\section{Theorem 4.2}

Let $\mathcal{Y}_{n}^{*}, \mathcal{Z}_{n}^{*}$ and $\mathcal{W}_{n}$ be defined as above. Then for any $\delta>0$, it holds that $\mathcal{Y}_{n}^{*}=\pi+n^{-5+\delta} o(1), \mathcal{Z}_{n}^{*}=\pi+$ $n^{-7+\delta} o(1), \mathcal{W}_{n}=\pi+n^{-9+\delta} o(1)$ and $n^{13 / 2}\left(\mathcal{Y}_{n}^{*}-\left(\pi-9 \pi^{7} /\left(2 n^{6}\right)\right)\right) \stackrel{\mathcal{L}}{\rightarrow} \mathcal{N}\left(0,136971 \pi^{14} / 2\right), n^{17 / 2}\left(\mathcal{Z}_{n}^{*}-(\pi-\right.$ $\left.\left.12 \pi^{9} / n^{8}\right)\right) \stackrel{\mathcal{L}}{\rightarrow} \mathcal{N}\left(0,6989472 \pi^{18}\right), n^{21 / 2}\left(\mathcal{W}_{n}-\left(\pi-180 \pi^{11} / n^{10}\right)\right) \stackrel{\mathcal{L}}{\rightarrow} \mathcal{N}\left(0,22852044000 \pi^{22}\right)$.

\section{Random Bisection}

Finally, we consider a variation of the above bisection process by allowing each newly added vertex $P_{i-1 / 2}$ between the two consecutive vertices $P_{i}$ and $P_{i-1}$ to be uniformly randomly chosen on arc $\widehat{P_{i-1} P_{i}}$, with each $P_{i-1 / 2}$ also independent of the others. For each newly added point $P_{i-1 / 2}$, the rescaled $X_{i-1 / 2}$ can now be written as $X_{i-1 / 2}=X_{i-1}+\left(X_{i}-X_{i-1}\right) U_{i}$ where $U_{1}, U_{2}, \cdots, U_{n}$ are independent and uniformly distributed over $(0,1)$ and are also independent of $X_{1}, X_{2}, \cdots, X_{n-1}$. The semiperimeters and areas of the newly constructed random inscribed and circumscribing $2 n$-gons can now respectively be expressed as $S_{2 n}=\sum_{i=1}^{n} \sin \left\{\pi\left(X_{i}-X_{i-1}\right) U_{i}\right\}+\sin \left\{\pi\left(X_{i}-X_{i-1}\right)\left(1-U_{i}\right)\right\}, A_{2 n}=\frac{1}{2} \sum_{i=1}^{n} \sin \left\{2 \pi\left(X_{i}-X_{i-1}\right) U_{i}\right\}+$ $\sin \left\{2 \pi\left(X_{i}-X_{i-1}\right)\left(1-U_{i}\right)\right\}, S_{2 n}^{\prime}=\sum_{i=1}^{n} \tan \left\{\pi\left(X_{i}-X_{i-1}\right) U_{i}\right\}+\tan \left\{\pi\left(X_{i}-X_{i-1}\right)\left(1-U_{i}\right)\right\}$.

Denote $\mathcal{M}_{n, k}=\sum_{i=1}^{n}\left|X_{i}-X_{i-1}\right|^{k}\left\{U_{i}^{k}+\left(1-U_{i}\right)^{k}\right\}$. Then, similar to (1), (2) and (3), we have, for any $m \geq 1$,

$$
\begin{aligned}
& S_{2 n}=\sum_{j=1}^{m} \frac{(-1)^{j-1}}{(2 j-1) !} \pi^{2 j-1} \mathcal{M}_{n, 2 j-1}+n^{-(2 m-1)+\delta} o(1), \\
& A_{2 n}=\sum_{j=1}^{m} \frac{(-1)^{j-1} 2^{2 j-2}}{(2 j-1) !} \pi^{2 j-1} \mathcal{M}_{n, 2 j-1}+n^{-(2 m-1)+\delta} o(1), \\
& S_{2 n}^{\prime}=\sum_{j=1}^{m} \frac{B_{2 j}(-4)^{j}\left(1-4^{j}\right)}{(2 j) !} \pi^{2 j-1} \mathcal{M}_{n, 2 j-1}+n^{-(2 m-1)+\delta} o(1) .
\end{aligned}
$$

Note that $U_{i}^{k}+\left(1-U_{i}\right)^{k}$ follows the same distribution as $\mathcal{D}_{2, k}$. Thus it is easy to compute that $\mathbb{E}\left(\mathcal{M}_{n, k}\right)=$ $\mathbb{E}\left(\mathcal{D}_{2, k}\right) \mathbb{E}\left(\mathcal{D}_{n, k}\right)=O\left(n^{-(k-1)}\right)$ and $\operatorname{Var}\left(\mathcal{M}_{n, k}\right)=\mathbb{E}\left(\mathcal{D}_{n, 2 k}\right) \operatorname{Var}\left(\mathcal{D}_{2, k}\right)=O\left(n^{-(2 k-1)}\right)$ for large $n$. 
Lemma 5.1 ([19])

Let $n, k \in \mathbb{N}$ and $\delta>0$. Then $n^{k-2-\delta} \mathcal{M}_{n, k} \rightarrow 0$ w.p.1 as $n \rightarrow \infty$ and $n^{k-1 / 2}\left(\mathcal{M}_{n, k}-k ! n^{-(k-1)} \mathbb{E}\left(\mathcal{D}_{2, k}\right)\right) \stackrel{\mathcal{L}}{\rightarrow}$ $\mathcal{N}\left(0, \widetilde{\sigma}_{k}^{2}\right)$ where $\widetilde{\sigma}_{k}^{2}=(2 k) ! \mathbb{E}\left(\mathcal{D}_{2, k}^{2}\right)-\left(1+k^{2}\right)(k !)^{2} \mathbb{E}\left(\mathcal{D}_{2, k}\right)^{2}, \mathbb{E}\left(\mathcal{D}_{2, k}\right)=\frac{2}{k+1}$, and $\mathbb{E}\left(\mathcal{D}_{2, k}^{2}\right)=\frac{2}{2 k+1}\left(1+\frac{(k !)^{2}}{(2 k) !}\right)$.

The next lemma establishes asymptotic estimates for general linear combinations of $\mathcal{M}_{n, k}$ and $\mathcal{D}_{n, k}$ and will be proved later in Section 6.

\section{Lemma 5.2}

Let $\mathcal{M}_{n, k}^{*}=\lambda_{1} \mathcal{M}_{n, k}+\lambda_{2} \mathcal{D}_{n, k}$ where $\lambda_{1}, \lambda_{2} \in \mathbb{R}$. Then $n^{k-2-\delta}\left(\mathcal{M}_{n, k}^{*}-\pi\right) \rightarrow 0$ w.p. 1 as $n \rightarrow \infty$ for any $\delta>0$ and $n^{k-1 / 2}\left\{\mathcal{M}_{n, k}^{*}-\left(\frac{2}{k+1} \lambda_{1}+\lambda_{2}\right) k ! / n^{k-1}\right\} \stackrel{\mathcal{L}}{\rightarrow} \mathcal{N}\left(0, \bar{\sigma}_{k}^{2}\right)$ where $\bar{\sigma}_{k}^{2}=\lambda_{1}^{2} \widetilde{\sigma}_{k}^{2}+\lambda_{2}^{2} \sigma_{k}^{2}+\frac{4}{k+1} \lambda_{1} \lambda_{2} \sigma_{k}^{2}$ and $\sigma_{k}^{2}=$ $(2 k) !-\left(1+k^{2}\right)(k !)^{2}$.

In particular, for $k=3$, we have from Lemma 5.1, $\mathcal{M}_{n, 3}=n^{-1+\delta} o(1)$ and $n^{5 / 2}\left(\mathcal{M}_{n, 3}-3 / n^{2}\right) \stackrel{\mathcal{L}}{\rightarrow} \mathcal{N}(0,126)$. Since $S_{2 n}=\pi-\frac{1}{3 !} \pi^{3} \mathcal{M}_{n, 3}+n^{-3+\delta} o(1), \quad A_{2 n}=\pi-\frac{4}{3 !} \pi^{3} \mathcal{M}_{n, 3}+n^{-3+\delta} o(1) \quad$ and $\quad S_{2 n}^{\prime}=\pi+\frac{1}{3} \pi^{3} \mathcal{M}_{n, 3}+$ $n^{-3+\delta} o(1)$, it follows that $n^{1-\delta}\left(S_{2 n}-\pi\right) \rightarrow 0, n^{1-\delta}\left(A_{2 n}-\pi\right) \rightarrow 0, n^{1-\delta}\left(S_{2 n}^{\prime}-\pi\right) \rightarrow 0$ w.p. 1 as $n \rightarrow \infty$ for any $\delta>0$. By Slutsky's theorem, we obtain immediately, $n^{5 / 2}\left(S_{2 n}-\left(\pi-\pi^{3} /\left(2 n^{2}\right)\right)\right) \stackrel{\mathcal{L}}{\rightarrow} \mathcal{N}\left(0,7 \pi^{6} / 2\right), n^{5 / 2}\left(A_{2 n}-\right.$ $\left.\left(\pi-2 \pi^{3} / n^{2}\right)\right) \stackrel{\mathcal{L}}{\rightarrow} \mathcal{N}\left(0,56 \pi^{6}\right)$ and $n^{5 / 2}\left(S_{2 n}^{\prime}-\left(\pi+\pi^{3} / n^{2}\right)\right) \stackrel{\mathcal{L}}{\rightarrow} \mathcal{N}\left(0,14 \pi^{6}\right)$.

To construct extrapolation improvements of $\pi$ based on the semiperimeters and areas of both the random $n$-gons and the newly constructed random $2 n$-gons, we again start with linear combinations of $S_{2 n}^{\prime}, S_{n}^{\prime}$ in the form of $\mathcal{X}_{n}^{\prime}=\alpha S_{2 n}^{\prime}+\beta S_{n}^{\prime}$ with $\alpha+\beta=1$. Using $S_{2 n}^{\prime}=\pi+\frac{1}{3} \pi^{3} \mathcal{M}_{n, 3}+n^{-3+\delta} o(1), S_{n}^{\prime}=\pi+\frac{1}{3} \pi^{3} \mathcal{D}_{n, 3}+n^{-3+\delta} o(1)$, we obtain $\mathcal{X}_{n}^{\prime}=\pi+\frac{1}{3} \pi^{3}\left(\alpha \mathcal{M}_{n, 3}+\beta \mathcal{D}_{n, 3}\right)+n^{-3+\delta} o(1)$ where $\alpha \mathcal{M}_{n, 3}+\beta \mathcal{D}_{n, 3}=\sum_{i=1}^{n}\left|X_{i}-X_{i-1}\right|^{3}\left\{\alpha\left[U_{i}^{3}+\right.\right.$ $\left.\left(1-U_{i}\right)^{3}\right]+\beta$ \}. Here again, there is no way to eliminate the leading error term $\alpha \mathcal{M}_{n, 3}+\beta \mathcal{D}_{n, 3}$. Consequently, we choose $\alpha$ and $\beta$ such that $\mathbb{E}\left(\alpha \mathcal{M}_{n, 3}+\beta \mathcal{D}_{n, 3}\right)=0$. This yields $\alpha=2, \beta=-1$ and thus $\mathcal{X}_{n}^{\prime}=2 S_{2 n}^{\prime}-S_{n}^{\prime}=$ $\pi+\frac{1}{3} \pi^{3} \mathcal{M}_{n, 3}^{*}+n^{-3+\delta} o(1)$ where $\mathcal{M}_{n, 3}^{*}=2 \mathcal{M}_{n, 3}-\mathcal{D}_{n, 3}$. Similarly, by combining $S_{2 n}^{\prime}$ with $S_{n}$, or $S_{2 n}^{\prime}$ with $A_{n}$, we can obtain $\mathcal{Y}_{n}^{\prime}=\frac{1}{2} S_{2 n}^{\prime}+\frac{1}{2} S_{n}=\pi+\frac{1}{12} \pi^{3} \mathcal{M}_{n, 3}^{*}+n^{-3+\delta} o(1)$ and $\mathcal{Z}_{n}^{\prime}=\frac{4}{5} S_{2 n}^{\prime}+\frac{1}{5} A_{n}=\pi+\frac{2}{15} \pi^{3} \mathcal{M}_{n, 3}^{*}+$ $n^{-3+\delta} o(1)$. By Lemma 5.2, we have $n^{5 / 2} \mathcal{M}_{n, 3}^{*} \stackrel{\mathcal{L}}{\rightarrow} \mathcal{N}(0,144)$. Then by Slutsky's theorem, we obtain $n^{5 / 2}\left(\mathcal{X}_{n}^{\prime}-\right.$ $\pi) \stackrel{\mathcal{L}}{\rightarrow} \mathcal{N}\left(0,16 \pi^{6}\right), n^{5 / 2}\left(\mathcal{Y}_{n}^{\prime}-\pi\right) \stackrel{\mathcal{L}}{\rightarrow} \mathcal{N}\left(0, \pi^{6}\right)$ and $n^{5 / 2}\left(\mathcal{Z}_{n}^{\prime}-\pi\right) \stackrel{\mathcal{L}}{\rightarrow} \mathcal{N}\left(0,64 \pi^{6} / 25\right)$. Therefore, we have

\section{Theorem 5.1}

Let $\mathcal{X}_{n}^{\prime}=2 S_{2 n}^{\prime}-S_{n}^{\prime}, \mathcal{Y}_{n}^{\prime}=\frac{1}{2} S_{2 n}^{\prime}+\frac{1}{2} S_{n}$ and $\mathcal{Z}_{n}^{\prime}=\frac{4}{5} S_{2 n}^{\prime}+\frac{1}{5} A_{n}$. Then for any $\delta>0$, it holds that $\mathcal{X}_{n}^{\prime}=$ $\pi+n^{-1+\delta} o(1), \mathcal{Y}_{n}^{\prime}=\pi+n^{-1+\delta} o(1), \mathcal{Z}_{n}^{\prime}=\pi+n^{-1+\delta} o(1)$ and $n^{5 / 2}\left(\mathcal{X}_{n}^{\prime}-\pi\right) \stackrel{\mathcal{L}}{\rightarrow} \mathcal{N}\left(0,16 \pi^{6}\right), n^{5 / 2}\left(\mathcal{Y}_{n}^{\prime}-\pi\right) \stackrel{\mathcal{L}}{\rightarrow}$ $\mathcal{N}\left(0, \pi^{6}\right), n^{5 / 2}\left(\mathcal{Z}_{n}^{\prime}-\pi\right) \stackrel{\mathcal{L}}{\rightarrow} \mathcal{N}\left(0,64 \pi^{6} / 25\right)$.

Similarly, for $\alpha+\beta=1$, we have $\mathcal{Y}_{n}^{*}=\alpha \mathcal{Y}_{2 n}+\beta \mathcal{Y}_{n}=\pi+\frac{\pi^{5}}{20}\left(\alpha \mathcal{M}_{n, 5}+\beta \mathcal{D}_{n, 5}\right)+n^{-5+\delta} o(1)$. By setting $\mathbb{E}\left(\alpha \mathcal{M}_{n, 5}+\beta \mathcal{D}_{n, 5}\right)=0$, we get $\alpha=3 / 2, \beta=-1 / 2$ so that $\mathcal{Y}_{n}^{*}=\frac{3}{2} \mathcal{Y}_{2 n}-\frac{1}{2} \mathcal{Y}_{n}=-\frac{1}{3} S_{n}-\frac{1}{6} S_{n}^{\prime}+S_{2 n}+\frac{1}{2} S_{2 n}^{\prime}=$ $\pi+\frac{1}{20} \pi^{5} \mathcal{M}_{n, 5}^{*}+n^{-5+\delta} o(1)$ where $\mathcal{M}_{n, 5}^{*}=\frac{3}{2} \mathcal{M}_{n, 5}-\frac{1}{2} \mathcal{D}_{n, 5}$ satisfies $n^{9 / 2} \mathcal{M}_{n, 5}^{*} \stackrel{\mathcal{L}}{\rightarrow} \mathcal{N}(0,583200)$.

Finally, we combine $\mathcal{Z}_{n}$ and $\mathcal{Z}_{2 n}$ to obtain $\mathcal{Z}_{n}^{*}=\frac{4}{3} \mathcal{Z}_{2 n}-\frac{1}{3} \mathcal{Z}_{n}=-\frac{16}{45} S_{n}+\frac{1}{15} A_{n}-\frac{2}{45} S_{n}^{\prime}+\frac{64}{45} S_{2 n}-$ $\frac{4}{15} A_{2 n}+\frac{8}{45} S_{2 n}^{\prime}=\pi+\frac{\pi^{7}}{105} \mathcal{M}_{n, 7}^{*}+n^{-7+\delta} o(1) \quad$ where $\quad \mathcal{M}_{n, 7}^{*}=-\frac{1}{3} \mathcal{D}_{n, 7}+\frac{4}{3} \mathcal{M}_{n, 7} \quad$ satisfies $\quad n^{13 / 2} \mathcal{M}_{n, 7}^{*} \stackrel{\mathcal{L}}{\rightarrow}$ $\mathcal{N}(0,10984028160)$. Therefore, we conclude as follows.

\section{Theorem 5.2}

Let $\mathcal{Y}_{n}^{*}$ and $\mathcal{Z}_{n}^{*}$ be defined as above. Then for any $\delta>0$, it holds that $\mathcal{Y}_{n}^{*}=\pi+n^{-3+\delta} o(1), \mathcal{Z}_{n}^{*}=\pi+n^{-5+\delta} o(1)$ and $n^{9 / 2}\left(\mathcal{Y}_{n}^{*}-\pi\right) \stackrel{\mathcal{L}}{\rightarrow} \mathcal{N}\left(0,1458 \pi^{10}\right), n^{13 / 2}\left(\mathcal{Z}_{n}^{*}-\pi\right) \stackrel{\mathcal{L}}{\rightarrow} \mathcal{N}\left(0,14944256 \pi^{14} / 15\right)$.

\section{Proof of Lemma 5.2}

The proof of Lemma 5.2 follows similar ideas as in $[2,19]$. Using the equivalent representation

$$
\left(X_{1}-X_{0}, X_{2}-X_{1}, \ldots, X_{n}-X_{n-1}\right) \stackrel{\mathcal{L}}{=}\left(\frac{V_{1}}{\sum_{i=1}^{n} V_{i}}, \frac{V_{2}}{\sum_{i=1}^{n} V_{i}}, \ldots, \frac{V_{n}}{\sum_{i=1}^{n} V_{i}}\right)
$$


where $V_{1}, V_{2}, \ldots, V_{n}$ are independent standard exponential random variables, we can rewrite

$$
\mathcal{M}_{n, k}^{*}=\lambda_{1} \mathcal{M}_{n, k}+\lambda_{2} \mathcal{D}_{n, k}=\frac{\sum_{i=1}^{n} V_{i}^{k}\left\{\lambda_{1}\left[U_{i}^{k}+\left(1-U_{i}^{k}\right)\right]+\lambda_{2}\right\}}{\left(\sum_{i=1}^{n} V_{i}\right)^{k}} .
$$

By the multivariate central limit theorem, we can now obtain, as $n \rightarrow \infty$,

$$
\sqrt{n}\left(\left(\begin{array}{c}
\frac{1}{n} \sum_{i=1}^{n} V_{i}^{k}\left\{\lambda_{1}\left[U_{i}^{k}+\left(1-U_{i}^{k}\right)\right]+\lambda_{2}\right\} \\
\frac{1}{n} \sum_{i=1}^{n} V_{i}
\end{array}\right)-\left(\begin{array}{c}
\mu_{1}^{*} \\
\mu_{2}^{*}
\end{array}\right)\right) \stackrel{\mathcal{L}}{\rightarrow} \mathcal{N}\left(0, \Sigma^{*}\right)
$$

where $\quad \mu_{1}^{*}=\mathbb{E}\left(V_{i}^{k}\left\{\lambda_{1}\left[U_{i}^{k}+\left(1-U_{i}^{k}\right)\right]+\lambda_{2}\right\}\right)=k !\left(\lambda_{1} \mathbb{E}\left(\mathcal{D}_{2, k}\right)+\lambda_{2}\right), \quad \mu_{2}^{*}=\mathbb{E}\left(V_{i}\right)=1 \quad$ and $\quad \Sigma^{*} \quad$ is $\quad$ the covariance matrix of the random vector $\left(V_{i}^{k}\left\{\lambda_{1}\left[U_{i}^{k}+\left(1-U_{i}^{k}\right)\right]+\lambda_{2}\right\}, V_{i}\right)$ with $\Sigma_{11}^{*}=\operatorname{Var}\left(V_{i}^{k}\left\{\lambda_{1}\left[U_{i}^{k}+\right.\right.\right.$ $\left.\left.\left.\left(1-U_{i}^{k}\right)\right]+\lambda_{2}\right\}\right)=(2 k) ! \mathbb{E}\left[\left(\lambda_{1} \mathcal{D}_{2, k}+\lambda_{2}\right)^{2}\right]-(k !)^{2}\left(\lambda_{1} \mathbb{E}\left(\mathcal{D}_{2, k}\right)+\lambda_{2}\right)^{2}, \quad \Sigma_{22}^{*}=\operatorname{Var}\left(V_{i}\right)=1 \quad$ and $\quad \Sigma_{12}^{*}=\Sigma_{21}^{*}=$ $\operatorname{Cov}\left(V_{i}^{k}\left\{\lambda_{1}\left[U_{i}^{k}+\left(1-U_{i}^{k}\right)\right]+\lambda_{2}\right\}, V_{i}\right)=k \cdot k !\left(\lambda_{1} \mathbb{E}\left(\mathcal{D}_{2, k}\right)+\lambda_{2}\right)$.

Finally, we choose $f(x, y)=x y^{-k}$ with $\frac{\partial f}{\partial x}=y^{-k}, \frac{\partial f}{\partial y}=-k x y^{-k-1}$. Then we have $f\left(\frac{1}{n} \sum_{i=1}^{n} V_{i}^{k}\left\{\lambda_{1}\left[U_{i}^{k}+\right.\right.\right.$ $\left.\left.\left.\left(1-U_{i}^{k}\right)\right]+\lambda_{2}\right\}, \frac{1}{n} \sum_{i=1}^{n} V_{i}\right)=n^{k-1} \mathcal{M}_{n, k}^{*}$ with $f\left(\mu_{1}^{*}, \mu_{2}^{*}\right)=\left(\frac{2}{k+1} \lambda_{1}+\lambda_{2}\right) k !, \frac{\partial f}{\partial x}\left(\mu_{1}^{*}, \mu_{2}^{*}\right)=1$ and $\frac{\partial f}{\partial y}\left(\mu_{1}^{*}, \mu_{2}^{*}\right)=$ $-k k !\left(\frac{2}{k+1} \lambda_{1}+\lambda_{2}\right)$. Thus by Cramér's theorem [6], we have, as $n \rightarrow \infty$,

$$
\sqrt{n}\left(n^{k-1} \mathcal{M}_{n, k}^{*}-\left(\frac{2}{k+1} \lambda_{1}+\lambda_{2}\right) k !\right)=n^{k-1 / 2}\left(\mathcal{M}_{n, k}^{*}-\left(\frac{2}{k+1} \lambda_{1}+\lambda_{2}\right) k ! / n^{k-1}\right) \stackrel{\mathcal{L}}{\rightarrow} \mathcal{N}\left(0, \bar{\sigma}_{k}^{2}\right)
$$

where $\bar{\sigma}_{k}^{2}=\left(1,-k k !\left(\frac{2}{k+1} \lambda_{1}+\lambda_{2}\right)\right) \Sigma^{*}\left(1,-k k !\left(\frac{2}{k+1} \lambda_{1}+\lambda_{2}\right)\right)^{T}=\lambda_{1}^{2} \widetilde{\sigma}_{k}^{2}+\lambda_{2}^{2} \sigma_{k}^{2}+\frac{4}{k+1} \lambda_{1} \lambda_{2} \sigma_{k}^{2}$.

This finishes the proof of Lemma 5.2.

\section{Conclusions}

Given $n$ independent points uniformly distributed on the unit circle in $\mathbb{R}^{2}$, we studied in this paper the problem of developing optimal extrapolation improvements of random approximations of $\pi$ based on geometric quantities such as the semiperimeter and area of the corresponding random cyclic polygons. By simultaneously considering these random $n$-gons and suitably constructed random $2 n$-gons and optimizing over functionals of their semiperimeters and areas, we derived several new estimates of $\pi$ with faster convergence rates. In addition, we also proved that these extrapolation improvements are asymptotically normal as $n \rightarrow \infty$. It would be interesting to further extend such asymptotic convergence results to higher dimensional cases or for more general non-uniform or non-i.i.d. distributions.

\section{Acknowledgments}

Research supported in part by National Natural Science Foundation of China (No. 11471028, 11801018, 11831003), Beijing Natural Science Foundation (No. 1182004, 1192001, Z180007), Youth Backbone Individual Program of the Organization Department of Beijing Municipality (No. 2017000020124G052).

\section{REFERENCES}

1. P. Beckmann, A History of $\pi$, Fifth edition, The Golem Press, 1982.

2. C. Bélisle, On the polygon generated by $n$ random points on a circle, Statist. Probab. Lett., 81 (2011) 236-242.

3. P. Billingsley, Probability and Measure, Third edition, Wiley, 1995.

4. D. A. Darling, On a class of problems related to the random division of an interval, Ann. Math. Statistics, 24 (1953) $239-253$.

5. W. Feller, An Introduction to Probability Theory and its Applications, Vol. II, Wiley, 1966.

6. T. S. Ferguson, A Course in Large Sample Theory, Chapman \& Hall, New York, 1996. 
7. T. Hsing, On the asymptotic distribution of the area outside a random convex hull in a disk, Ann. Appl. Probab, 4 (1994), no. 2, 478-493.

8. I. Hueter, The convex hull of a normal sample, Adv. in Appl. Probab, 26 (1994), no. 4, 855-875.

9. D. C. Joyce, Survey of extrapolation processes in numerical analysis, SIAM Review, 13 (1971) 435-490.

10. T. Li, Essays on $\pi$ (Chinese), Higher Education Press, 2007.

11. R. Pyke, Spacings, J. Roy. Statist. Soc. Ser. B, 27 (1965) 395-449.

12. P. Rabinowitz, Extrapolation methods in numerical integration, Numer. Algorithms, 3 (1992) 17-28.

13. A. Rényi, R. Sulanke, Über die konvexe Hülle von n zufällig gewählten Punkten I, Z. Wahrscheinlichkeitstheorie und Verw. Gebiete, 2 (1963), 75-84.

14. A. Rényi, R. Sulanke, Über die konvexe Hülle von n zufällig gewählten Punkten II, Z. Wahrscheinlichkeitstheorie und Verw. Gebiete, 3 (1964), 138-147.

15. R. J. Serfling, Approximation Theorems of Mathematical Statistics, Wiley, 1980.

16. T. Tang, Brief Discussions on Computation Mathematics from the Calculations of $\pi$ (Chinese), Higher Education Press, 2018.

17. V. Vu, Central limit theorems for random polytopes in a smooth convex set, Adv. Math., 207 (2006), no. 1, 221-243.

18. S.-S. Wang and W.-Q. Xu, Random cyclic polygons from Dirichlet distributions and approximations of $\pi$, Statist. Probab. Lett., 140 (2018) 84-90.

19. W.-Q. Xu, Extrapolation methods for random approximations of $\pi$, J. Numer. Math. Stoch., 5 (2013) 81-92.

20. W.-Q. Xu, Random circumscribing polygons and approximations of $\pi$, Statist. Probab. Lett., 106 (2015) 52-57.

21. W.-Q. Xu, L. Meng and Y. Li, Random polygons and estimations of $\pi$, Open Math., 17 (2019) 575-581. 